\title{
COMPACTNESS IN $L_{\infty}$ SPACES
}

BARRY M. CHERKAS

Conditions for compactness in $L_{\infty}(S, \Sigma, \mu)$ are known when $\mu(S)$ is finite [1, p. 297]. The purpose of this note is to state a compactness criterion which does not depend on $\mu(S)$. The proof uses the standard diagonal procedure. It should be noted that the criterion is not a necessary one unless the underlying space $S$ is $\sigma$-finite (see remark at the end).

Preliminaries. We assume that $(S, \Sigma, \mu)$ is a positive measure space $[1$, p. 126]. The triple $(S, \Sigma, \mu)$ is said to be $\sigma$-finite whenever there is a sequence of sets in $\Sigma$ of finite $\mu$-measure whose union is $S$. A finite decomposition (of $S$ ) is a finite collection of pairwise disjoint sets in $\Sigma$ whose union is $S$. A set $N \in \Sigma$ is called locally $\mu$-null if $\mu(E \cap N)=0$ for all $E \in \Sigma$ such that $\mu(E)<\infty$. Let $\mathfrak{N}$ denote the family of all locally $\mu$-null sets. A complex-valued, $\mu$-measurable function $f$ on $S[1$, p. 106] is said to be $\mu$-essentially bounded if for some real number $a \geqq 0$ we have $\{s:|f(s)|>a\} \in \Re$. Let

$$
\|f\|_{\infty}=\inf \{a:\{s:|f(s)|>a\} \in \Re\} \text {. }
$$

We denote by $L_{\infty}^{0}(S, \Sigma, \mu)$ the space of all complex-valued, $\mu$-essentially bounded, $\mu$-measurable functions on $S . L_{\infty}^{0}(S, \Sigma, \mu)$ is partitioned according to the equivalence relation: $f$ is equivalent to $g$ if and only if $\|f-g\|_{\infty}=0$. Denote the class of all functions equivalent to $f$ by $[f]$ and let $L_{\infty}(S, \Sigma, \mu)$ denote the space of all such equivalence classes $[f] \subseteq L_{\infty}^{0}(S, \Sigma, \mu)$. With the induced norm $\|[f]\|_{\infty}=\|f\|_{\infty}$, $L_{\infty}(S, \Sigma, \mu)$ is a Banach space [2, p. 347].

Definition 1. For any $f \in L_{\infty}^{0}(S, \Sigma, \mu)$ and nonempty set $E \in \Sigma$ we define the $\mu$-essential height of $f$ on $E$ by the seminorm

$$
|f|_{E}=\inf \{\sup \{|f(s)-f(t)|: s, t \in E-N \cap E\}: N \in \mathfrak{N}\} .
$$

For convenience, we set $|f|_{E}=0$ when $E$ is the empty set.

Lemma. Suppose $(S, \Sigma, \mu)$ is $\sigma$-finite. For $f \in L_{\infty}^{0}(S, \Sigma, \mu)$ and any $\epsilon>0$ there corresponds a finite decomposition $\left\{E_{k}: k=1, \cdots, n\right\}$ such that

$$
\max \left\{|f|_{E_{k}}: k=1, \cdots, n\right\}<\epsilon .
$$

Proof. We first show this when $f$ is real-valued. Given $\epsilon>0$ we construct a decomposition of $S$ in the following way: For integers $k$

Received by the editors December 7, 1968. 
which satisfy $\epsilon-\|f\|_{\infty}<k \epsilon<\|f\|_{\infty}$, set

$$
G_{k}^{*}=\{s:(k-1) \epsilon<f(s) \leqq k \epsilon\} .
$$

For the integer $n$ which satisfies $(n-1) \epsilon \leqq\|f\|_{\infty}<n \epsilon$, set

$$
\begin{aligned}
G_{n}^{*} & =\left\{s:(n-1) \epsilon<f(s) \leqq\|f\|_{\infty}\right\}, \\
G_{-(n-1)}^{*} & =\left\{s:-\|f\|_{\infty} \leqq f(s) \leqq-(n-1) \epsilon\right\},
\end{aligned}
$$

and

$$
G_{-n}^{*}=\left\{s:|f(s)|>\|f\|_{\infty}\right\} .
$$

From $[2$, p. 347$]$ we have $G_{-n}^{*} \in \mathcal{N}$. Let $\left(S, \Sigma^{*}, \mu\right)$ be the Lebesgue extension of $(S, \Sigma, \mu)$ [1, p. 143]. From the $\sigma$-finite property and $[1$, p. 148$]$, for each $k, G_{k}^{*} \in \Sigma^{*}$. By definition [1, p. 142], for some $G_{k}^{\prime} \in \Sigma$ and $N_{k}^{\prime} \in \mathscr{N}$, we have $G_{k}{ }^{*}=M_{k} \cup G_{k}^{\prime}$ for some subset $M_{k}$ of $N_{k}^{\prime}$. Let

$$
G_{-n}=G_{-n}^{\prime} \cup\left(\bigcup_{k=-n}^{n} N_{k}^{\prime}\right) \text { and } G_{k}=G_{k}^{\prime}-G_{k}^{\prime} \cap G_{-n} .
$$

By construction, $\left\{G_{k}\right\}$ is pairwise disjoint and $\bigcup_{k=-n}^{n} G_{k}=S$. Thus, $\left\{G_{k}\right\}$ is a finite decomposition such that $|f|_{G_{k}}<\epsilon$ for each $k$. For a complex-valued function, we need only consider the intersections from the finite decompositions for the real and imaginary parts derived as above.

The following definition generalizes the notion of uniform equicontinuity [3, p. 239] quite naturally to families of functions in $L_{\infty}^{0}(S, \Sigma, \mu)$.

DEFinition 2. A family of complex-valued $\mu$-measurable functions, $\{f\}$, is quasi-uniformly equi- $\mu$-measurable (abbreviated que $\mu$-measurable) if and only if to each $\epsilon>0$ there corresponds a finite decomposition $\left\{E_{k}: k=1, \cdots, n\right\}$ such that on each $E_{k}$, the $\mu$-essential height of every $f \in\{f\}$ is not greater than $\epsilon$.

A representative set of $K \subseteq L_{\infty}(S, \Sigma, \mu)$ is any set $K_{r}$ in $L_{\infty}^{0}(S, \Sigma, \mu)$ which consists of one and only one function from each equivalence class in $K$. It is easy to verify that whenever $K \subseteq L_{\infty}(S, \Sigma, \mu)$ has one representative set which is que $\mu$-measurable then every representative set of $K$ is que $\mu$-measurable. Moreover, the existence of a representative set is assured by the Axiom of Choice. Thus, we may formulate a nontrivial definition of que $\mu$-measurability in $L_{\infty}(S, \Sigma, \mu)$ without ambiguity.

Definition 3. A subset $K$ of $L_{\infty}(S, \Sigma, \mu)$ is said to be que $\mu$-measurable if and only if every representative set of $K$ is que $\mu$-measurable. 
Theorem. $A$ bounded set $K$ in $L_{\infty}(S, \Sigma, \mu)$ is conditionally compact if $K$ is que $\mu$-measurable. The converse holds when $(S, \Sigma, \mu)$ is $\sigma$-finite.

Proof. (Necessity) Given $\epsilon>0$ there are elements $\left[f_{1}\right], \cdots,\left[f_{m}\right]$ in $K$ such that for any $[f] \in K$,

$$
\min \left\{\left\|[f]-\left[f_{j}\right]\right\|_{\infty}: j=1, \cdots, m\right\} \leqq \epsilon / 3 .
$$

Let $K_{r}$ denote any representative set for $K$ and denote by $f_{1}, \cdots, f_{m}$ the representatives in $K_{r}$ corresponding to $\left[f_{1}\right], \cdots,\left[f_{m}\right]$, respectively. Using the above lemma, it is not difficult to show that any finite set in $L_{\infty}^{0}(S, \Sigma, \mu)$ is que $\mu$-measurable. Thus, there is a finite decomposition $\left\{E_{k}: k=1, \cdots, n\right\}$ such that $\left|f_{j}\right|_{E_{k}} \leqq \epsilon / 3$ for $j$ $=1, \cdots, m$ and $k=1, \cdots, n$. For any $f \in K_{r}$ and $s, t \in E_{k}$ we have

$$
|f(s)-f(t)| \leqq\left|f(s)-f_{j}(s)\right|+\left|f_{j}(s)-f_{j}(t)\right|+\left|f_{j}(t)-f(t)\right| \text {. }
$$

Thus, $|f|_{E_{k}} \leqq 2\left|f-f_{j}\right|_{E_{k}}+\epsilon / 3$. Therefore, taking the minimum of both sides with respect to $j$ we have $|f|_{E_{k}} \leqq \epsilon$. Hence, $K_{r}$ is que $\mu$-measurable.

(Sufficiency) Since $K$ is bounded there is a constant $M$ such that $\sup \left\{\|[f]\|_{\infty}:[f] \in K\right\} \leqq M$. Select a representative set $K_{r}$ for $K$ in which every function $f$ satisfies $|f(s)| \leqq M$ for all $s \in S$. For any sequence $\left\{\left[f_{n}\right]\right\}$ in $K$ let $\left\{f_{n}\right\}$ be its representative sequence in $K_{r}$. To each $\epsilon_{k}=1 / k$ there corresponds a finite decomposition $\left\{E_{k, j}: j=1, \cdots, l_{k}\right\}$ such that

$$
\max \left\{\sup \left\{\left|f_{n}\right|_{E_{k, j}}: f_{n} \in\left\{f_{n}\right\}\right\}: j=1, \cdots, l_{k}\right\} \leqq \epsilon_{k} .
$$

For each $E_{k, j}$ there is $F_{k, j} \subseteq E_{k, j}$ such that $E_{k, j}-F_{k, j} \in \mathfrak{N}$ and

$$
\sup \left\{\left|f_{n}(s)-f_{n}(t)\right|: s, t \in F_{k, j} \text { and } f_{n} \in\left\{f_{n}\right\}\right\} \leqq \epsilon_{k} .
$$

This follows since a countable union of locally $\mu$-null sets is locally $\mu$-null. From each $F_{k, j}$ we select any element $s_{k, j}$. For a fixed $s_{k, j}$ the set $\left\{f_{n}\left(s_{k, j}\right): f_{n} \in\left\{f_{n}\right\}\right\}$ is a bounded set of complex numbers. Thus, by the Bolzano-Weierstrass theorem, for $k=1$ there is a subsequence $\left\{f_{1_{n}}\right\}$ of $\left\{f_{n}\right\}$ which converges uniformly at the points $\left\{s_{1, j}: j=1, \cdots, l_{1}\right\}$. For $k=2$, there is a subsequence $\left\{f_{2_{n}}\right\}$ of $\left\{f_{1_{n}}\right\}$ which converges uniformly at the points $\left\{s_{1, i}\right\} \cup\left\{s_{2, j}\right\}$. Continuing in this way, by the diagonal method we obtain a subsequence $\left\{f_{n_{n}}\right\}$ which converges at each point of the set $\left\{s_{k, j}: k=1, \cdots ; j=1, \cdots, l_{k}\right\}$ and the convergence is uniform on any finite subset.

Given $\epsilon>0$, choose $p$ such that $\epsilon_{p} \leqq \epsilon / 3$. Since $\left\{f_{n_{n}}\right\}$ converges uniformly on $\left\{s_{p, j}: j=1, \cdots, l_{p}\right\}$, there is some integer $N$ such that if $n, m \geqq N$ we have 


$$
\max \left\{\left|f_{n_{n}}\left(s_{p, j}\right)-f_{m_{m}}\left(s_{p, j}\right)\right|: j=1, \cdots, l_{p}\right\} \leqq \epsilon / 3 .
$$

For any $s \in F_{p, j}$ we have

$$
\begin{aligned}
\left|f_{n_{n}}(s)-f_{m_{m}}(s)\right| \leqq & \left|f_{n_{n}}(s)-f_{n_{n}}\left(s_{p, j}\right)\right|+\left|f_{n_{n}}\left(s_{p, j}\right)-f_{m_{m}}\left(s_{p, j}\right)\right| \\
& +\left|f_{m_{m}}\left(s_{p, j}\right)-f_{m_{m}}(s)\right| \\
\leqq & 2 \epsilon_{p}+\epsilon / 3 .
\end{aligned}
$$

Thus, $\left\|f_{n_{n}}-f_{m_{m}}\right\|_{\infty} \leqq \epsilon$ if $n, m \geqq N$. Therefore $\left\{\left[f_{n_{n}}\right]\right\}$ is a Cauchy sequence in $K$. Hence, $K$ is conditionally compact.

REMARK. If $(S, \Sigma, \mu)$ is not $\sigma$-finite, the above criterion for compactness is not a necessary one as the following example illustrates: Let $S=[0,1], \Sigma$ be the usual Borel field of sets in $S$, and $\mu$ be the counting measure which assigns to each set in $\Sigma$ its cardinality. Select any nonmeasurable set $D \subset S$ and let $f(s)=0$ for $s \in D$ and $f(s)=1$ on $S-D$. From [1, p. 148], $f$ is $\mu$-measurable. However, $f$ does not satisfy Definition 2 for any $\epsilon<1$. Thus, the set $\{[f]\}$, although obviously compact in $L_{\infty}([0,1], \Sigma, \mu)$, is not que $\mu$-measurable.

Acknowledgement. The author wishes to thank Professor C. T. Taam for suggesting this area of investigation and also for his valuable comments on the problem.

\section{REFERENCES}

1. N. Dunford and J. Schwartz, Linear operators, I: General theory, Pure and Appl. Math., vol. 7, Interscience, New York, 1958. MR 22 \#8302.

2. E. Hewitt and K. Stromberg, Real and abstract analysis, A modern treatment of the theory of functions of a real variable, Springer-Verlag, Berlin and New York, 1965. MR $32 \# 5826$.

3. J. L. Kelley, General topology, Van Nostrand, Princeton, N.J., 1955. MR 16, 1136.

Hunter College, City University of New York, New York, New York 10021 